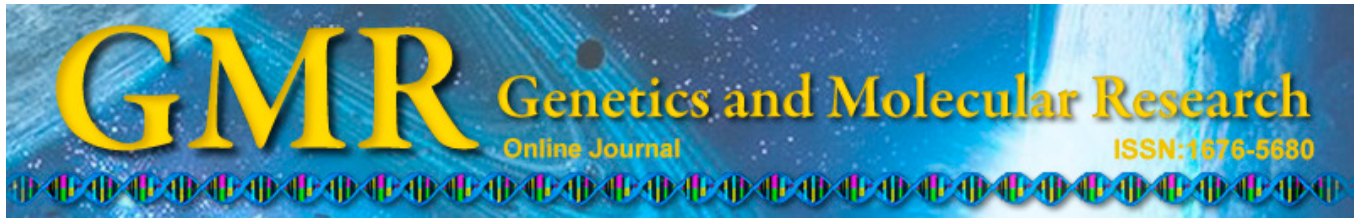

\title{
S-phase kinase-associated protein 2 expression interference inhibits breast cancer cell proliferation
}

\author{
Y.J. Sun, X.K. Wang and B.J. Li \\ Department of Pathology, Tianjin Fifth Central Hospital, Tanggu, \\ Tianjin, China \\ Corresponding author: Y.J. Sun \\ E-mail: Yajingsun189@126.com
}

Genet. Mol. Res. 14 (3): 9244-9252 (2015)

Received October 31, 2014

Accepted April 6, 2015

Published August 10, 2015

DOI http://dx.doi.org/10.4238/2015.August.10.4

\begin{abstract}
We investigated the expression of S-phase kinaseassociated protein 2 (SKP2) in breast cancer tissues, and the effects of $S K P 2$-specific small interfering RNA (siRNA) interference on breast cancer cell proliferation. Thirty subjects provided breast cancer tissue samples and 18 subjects provided normal breast specimens for this study. The expression of SKP2 in breast cancer patient tissues and normal breast tissues was detected by western blotting analysis and reverse transcription-polymerase chain reaction. SKP2specific siRNA was used to decrease SKP2 expression in breast cancer cell line MDA-MB-231. The 3-(4,5-dimethylthiazol-2-yl)2,5-diphenyltetrazolium bromide (MTT) assay was used to detect cell proliferation. SKP2 expression in breast cancer tissues was significantly higher than in normal breast tissues $(\mathrm{P}<0.05)$. Two pairs of siRNA specific to SKP2 were required to downregulate SKP2 expression in the breast cancer cell line MDA-MB-231. The MTT assay showed that MDA-MB-231 growth significantly slowed after SKP2 interference. Patients with breast cancer have an increased
\end{abstract}


SKP2 level. Interference in $S K P 2$ gene expression can inhibit breast cancer cell growth, suggesting that SKP2 is potentially a new target for breast cancer therapy.

Key words: Breast cancer; S-phase kinase-associated protein 2; SKP2; siRNA interference

\section{INTRODUCTION}

Breast cancer is a common tumor that threatens women's health. It is usually caused by malignant proliferation of mammary gland cells or acinar cells (Deapen, 2007). The incidence of breast cancer has risen markedly in most countries recently. Distal metastasis of breast cancer is the main cause of death (Njor et al., 2012; Meads and Moore, 2013). In a clinical context, breast cancer usually requires surgery, chemotherapy, radiotherapy, endocrine therapy, hormone therapy, biological target therapy, etc. (Brown-Glaberman et al., 2014). However, there is still no effective way to cure breast cancer (Al Joudi, 2014; Cho et al., 2014). Therefore, exploring the mechanism of breast cancer and searching for an appropriate gene therapy is the focus of research into the treatment of the disorder.

Tumorigenesis results in malignant cell proliferation, and involves a long and complex process of single or multiple gene mutation arising from chromosomal damage in the normal cell (Polak et al., 2014). Proto-oncogene activation, tumor suppressor gene inactivation, or out-of-control DNA transcription causes disruption to the cell cycle regulation mechanism, resulting in malignant cell proliferation (Solyom et al., 2012). Therefore, cell cycle regulation is vital for cell proliferation and tumorigenesis. The cell cycle involves cell cycle proteins (cyclins) and cyclin-dependent kinase (CDK) enzymes that positively regulate cell cycle progression, while cyclin-dependent kinase inhibitor (CKI) plays a negative regulatory role (Stivala et al., 2012; Jiang et al., 2014). Interaction between the two components controls the cell cycle process. It has been found that S-phase kinase-associated protein 2 (SKP2), a member of the F-box family with a gene located in the short arm of chromosome 5 (5p13), can interact with the S-phase cyclin-dependent kinase A-CDK2 (Zhang et al., 1995). SKP2 constitutes one of the subunits of the ubiquitin protein ligase complex SCF-SKP2, which can specifically identify phosphorylated substrates and mediate ubiquitin degradation (Chen et al., 2008). SKP2 mainly mediates the ubiquitin degradation of CDK inhibitors, such as $\mathrm{p} 21^{\mathrm{cip} 1}, \mathrm{p} 27^{\mathrm{kip} 1}$, and $\mathrm{p} 57^{\mathrm{kip} 2}$. Its expression is quite low in the G0/G1 phase, but increases in the S phase. Overexpressed SKP2 in the G0/G1 phase leads to an out-ofcontrol G1/S phase, disruption of cell proliferation, and differentiation (Mamillapalli et al., 2001; Hao et al., 2005). Therefore, SKP2 is a type of proto-oncogene, and its activation can promote the occurrence of tumors.

Recent studies have shown that $S K P 2$ expression can promote the progress of various cancers, while $S K P 2$ knock-out can stabilize CKIs resulting in cell cycle stagnation or delay (Shibahara et al., 2005; Chen et al., 2014). However, the mechanism by which SKP2 promotes the occurrence and development of breast cancer is still unclear. In the present study, we detected SKP2 expression in breast cancer tissue and investigated its role in cancer cell growth to find an effective therapeutic target for breast cancer and elucidate the mechanism by which it works. 


\section{MATERIAL AND METHODS}

\section{General information}

Thirty patients with invasive breast cancer provided tissue samples and 18 patients provided normal breast specimens between May 2012 and October 2014. The breast cancer patients included: 12 cases in stage I+IIA, 10 cases in stage IIB+III, and 8 cases in stage II+III. All the cancer patients were women and their mean age was $56.7 \pm 15.6$ years. All patients received surgical treatment and the specimens were diagnosed by pathological examination. The average age of patients in the normal control group was $48.6 \pm 13.2$ years. There was no statistically significant difference in gender and age distribution between the two groups. Specimens from patients with related diseases such as mastitis were excluded. All patients provided written informed consent. This study was approved by the Ethics Committee.

\section{Experiment reagents}

Dulbecco's modified Eagle's medium (DMEM) and fetal bovine serum were bought from Hyclone Co., Ltd. (Logan, UT, USA). L-glutamine and penicillin streptomycin were from Gibco Co., Ltd. (Carlsbad, CA, USA). SKP2 siRNA and control siRNA were designed and synthesized by the GenePharma Biological Co., Ltd. (Shanghai, China). The two SKP2 siRNA synthesis sequences were as follows: siRNA1-F: 5'-tcgaGGGAGTGACAAAGACTT TGgagtactgCAAAGTCTTTGTCACTCCCTTTTT-3'; siRNA1-R: 5'-ctagAAAAAGGGAGT GACAAAGACTTTGcagtactcCAAAGTCTTTGTCACTCCC-3'; and siRNA2-F: 5'-AGCTT TTCCAAAAAAGGGAGTGACAAAGACTTTGTCTCTTGAACAAAGTCTTTGTCACT CCCG-3'; siRNA2-R: 5'-GATCCGGGAGTGACAAAGACTTTGTTCAAGAGACAAAGT CTTTGTCACTCCCTTTTTTGGAAA-3'. Lipofectamine 2000 was bought from Invitrogen Co., Ltd. (Carlsbad, CA, USA). SKP2, $\beta$-actin antibodies, and horseradish peroxidase secondary antibody were purchased from Santa Cruz Co., Ltd. (Dallas, TX, USA). An RNA extraction kit and a reverse transcription-polymerase chain reaction (RT-PCR) kit were purchased from Promega Co., Ltd. (Madison, WI, USA).

\section{Cell culture}

The breast cancer cell line MDA-MB-231 was maintained in DMEM, with $100 \mathrm{mM}$ L-glutamine, $10 \%$ fetal bovine serum, and $1 \%$ penicillin streptomycin in a humid atmosphere containing $5 \% \mathrm{CO}_{2}$ at $37^{\circ} \mathrm{C}$. Cells in the logarithmic phase of growth were used for all experiments.

\section{Cell transfection}

SKP2 siRNA (Shanghai GenePharma Co., Ltd., China) was transfected into $2 \times 10^{5}$ MDA-MB-231 cells using Lipofectamine 2000 reagent according to the manufacturer instructions. Cells transfected with the transfection agent and scramble-control siRNA (negative control) were used as controls. After maintaining in the transfection medium for $6 \mathrm{~h}$, the medium was changed to DMEM and cells were cultured for the required time. 


\section{Real-time RT-PCR}

Real-time RT-PCR was used to verify differential expression of SKP2. The cDNA was synthesized using reverse transcriptase (TaKaRa), and oligo (dT) primers with $1 \mu \mathrm{g}$ RNA from the samples. The primers used were as follows: SKP2: forward primer: 5'-GCTGCTAAAGGTCTCTGGTGT-3' and reverse primer: 5'-AGGCTTAGATTCTGCAACTTG-3'; $\beta$-actin: forward primer: 5'-TGGCACCCAGCACAATGAA-3' and reverse primer: 5'-TAAGTCATAGTCCGCCTAGAAGCA-3'.

Each real-time RT-PCR mixture (in $20 \mu \mathrm{L}$ ) contained 2.5X SYBR Green Real-Time PCR Master Mix (TIANGEN), $0.5 \mu \mathrm{M}$ primers, and $0.5 \mu \mathrm{L}$ template cDNA. The cycling conditions consisted of an initial, single cycle of $15 \mathrm{~s}$ at $95^{\circ} \mathrm{C}$, followed by 40 cycles of $5 \mathrm{~s}$ at $95^{\circ} \mathrm{C}, 30 \mathrm{~s}$ at $58^{\circ} \mathrm{C}$, and $5 \mathrm{~s}$ at $72^{\circ} \mathrm{C}$. PCR amplifications were performed in three duplicates for each sample. Gene expression levels were quantified relative to the expression of $\beta$-actin by measuring the optical density at $490 \mathrm{~nm}\left(\mathrm{OD}_{490}\right)$. The differences in gene expression levels between groups were compared using the Student $t$-test. P values $<0.05$ were considered to be statistically significant.

\section{MTT assay}

Cells were seeded on 48-well plates at a density of $5 \times 10^{5}$ cells/well and incubated overnight at $37^{\circ} \mathrm{C}$. The cells were transfected with siRNA1 and siRNA2 of SKP2 and the negative control. The cells were then incubated for different time at $37^{\circ} \mathrm{C}$. After addition of $20 \mu \mathrm{L} 5 \mathrm{mg} / \mathrm{mL}$ MTT to each well, the plates were incubated for $4 \mathrm{~h}$ at $37^{\circ} \mathrm{C}$. Dimethyl sulfoxide $(300 \mu \mathrm{L})$ was added to each well and the absorbance at $490 \mathrm{~nm}$ was measured using a spectrophotometer.

\section{Western blot analysis}

The tissues were digested and homogenized with lysis buffer. Total protein was separated by denaturing $10 \%$ sodium dodecyl sulfate polyacrylamide gel electrophoresis. Detection was performed using a Quantity One system. Antibody dilutions were 1:1000 for SKP2 and 1:5000 for $\beta$-actin. Protein levels were normalized to $\beta$-actin and changes were determined.

\section{RESULTS}

\section{SKP2 is overexpressed in breast cancer tissue}

The breast cancer tissues were confirmed by hematoxylin and eosin staining (Figure 1). Western blotting was used to detect SKP2 expression in breast cancer tissue samples and normal breast tissue. The average OD, determined by the Quantity One system, in the breast cancer specimens was compared with that in the normal mammary gland samples. SKP2 expression in breast cancer tissue was significantly higher than in the normal controls (Figure $2 \mathrm{~A})$. The statistical results are shown in Figure $2 \mathrm{~B}(\mathrm{P}<0.01)$. 

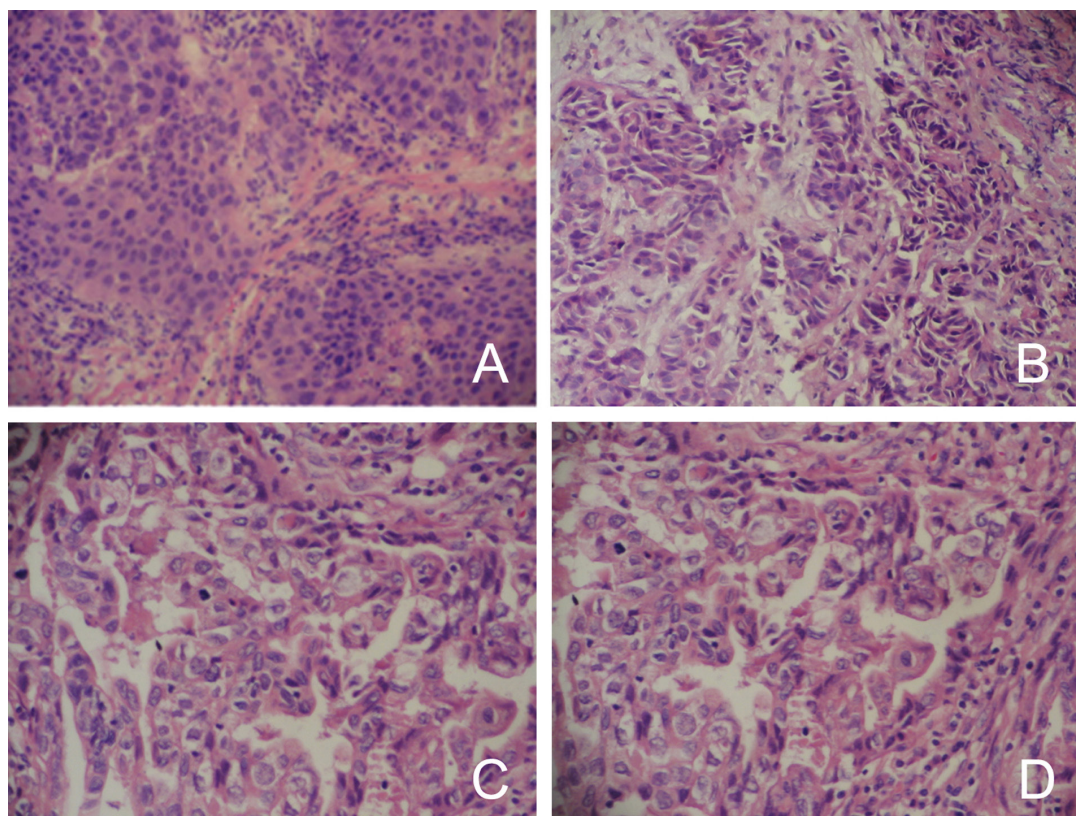

Figure 1. Breast cancer tissue hematoxylin and eosin staining (400X). A. B. Invasive breast cancer; C. D. invasive ductal carcinoma.
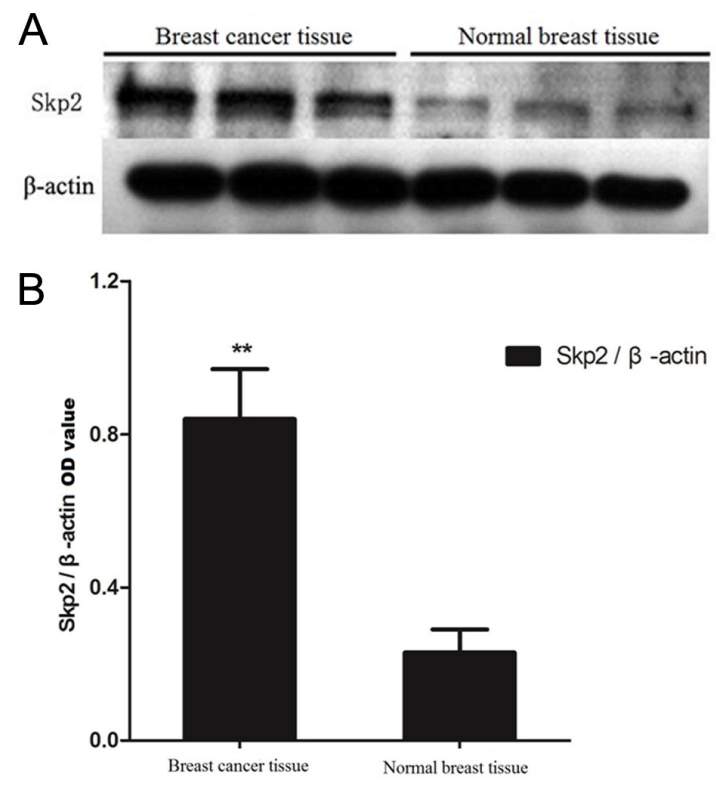

Figure 2. SKP2 expression in breast cancer tissue and normal breast tissue. A. Western blotting was used to detect SKP2 expression; B. SKP2 optical density (OD) value of breast cancer tissue and normal breast tissue. ${ }^{*} \mathrm{P}<0.01$. 


\section{SKP2 expression after siRNA treatment in MDA-MB-231}

To test whether SKP2 is an effective therapeutic target for breast cancer cells, we designed two pairs of the specificity endogenous interference for SKP2. After transfection with siRNA1 and siRNA2 for $48 \mathrm{~h}$, western blotting was used to detect endogenous SKP2 expression in MDA-MB-231 (Figure 3). siRNA1 and siRNA2 can both disturb endogenous SKP2 expression in breast cancer cells, but siRNA1 had a greater effect. MDA-MB-231 without any treatment were used as the negative control.

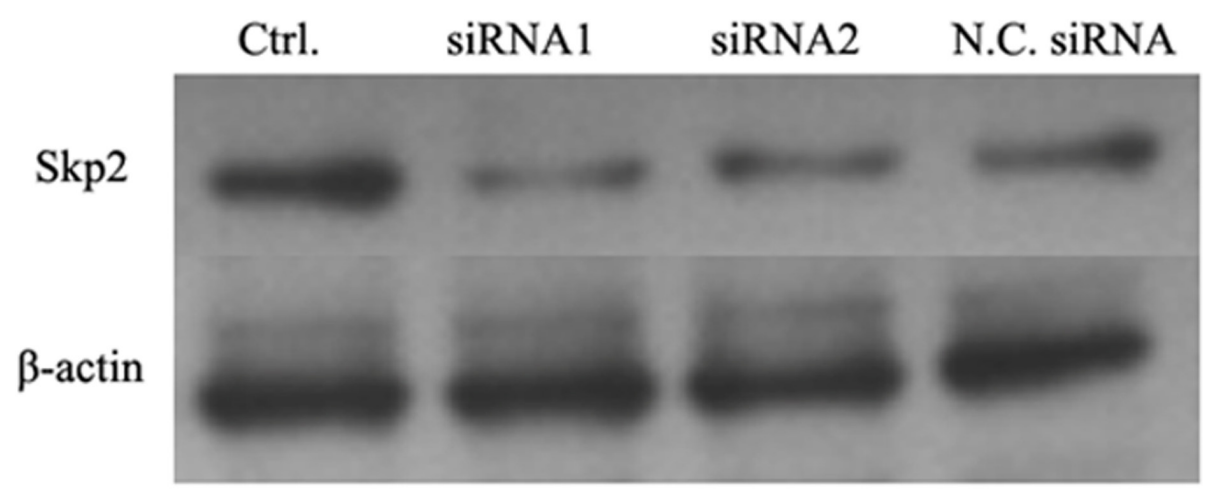

Figure 3. SKP2 siRNA effect. After transfection with siRNA for $48 \mathrm{~h}$, SKP2 expression was detected by western blotting. N.C. siRNA was used as a negative control.

\section{siRNA SKP2 inhibits MDA-MB-231 proliferation}

To test the effect of SKP2 interference on breast cancer cell proliferation, an MTT assay was applied. The results showed that after transfection of $S K P 2$ siRNA for 48, 72, and $96 \mathrm{~h}$, the $\mathrm{OD}_{490}$ value in the SKP2 siRNA group was significantly lower than in the negative control siRNA group (Figure 4). This indicated that the cell proliferation capability of the cells transfected with SKP2 siRNA was significantly weaker than in those cells transfected with negative control. siRNA or normal control cells. The cell proliferation in each group after transfection for 24 h showed no significant difference $(\mathrm{P}>0.05)$, while the cell proliferation speed slowed down after transfection of SKP2 siRNA for $48 \mathrm{~h}(\mathrm{P}<0.05)$ compared with the control group. After transfection of siRNA for 72 and $96 \mathrm{~h}$, the $\mathrm{OD}_{490}$ of the SKP2 siRNA group was significantly lower than in the negative control siRNA group $(\mathrm{P}<0.01)$, which indicated that downregulating $S K P 2$ expression can significantly inhibit the proliferation capability of the breast cancer cells.

\section{DISCUSSION}

Breast cancer is one of the most common malignant tumors in females. Its incidence is growing at $2 \%$ a year. About 1.2 million women develop, and 500,000 women die from breast cancer worldwide every year (Goldner et al., 2014; Keramatinia et al., 2014). It accounts for $7-10 \%$ of malignant tumors, second only in women patients to ovarian cancer. Breast cancer often occurs in the mammary gland epithelial tissue, where it poses the greatest threat to wom- 


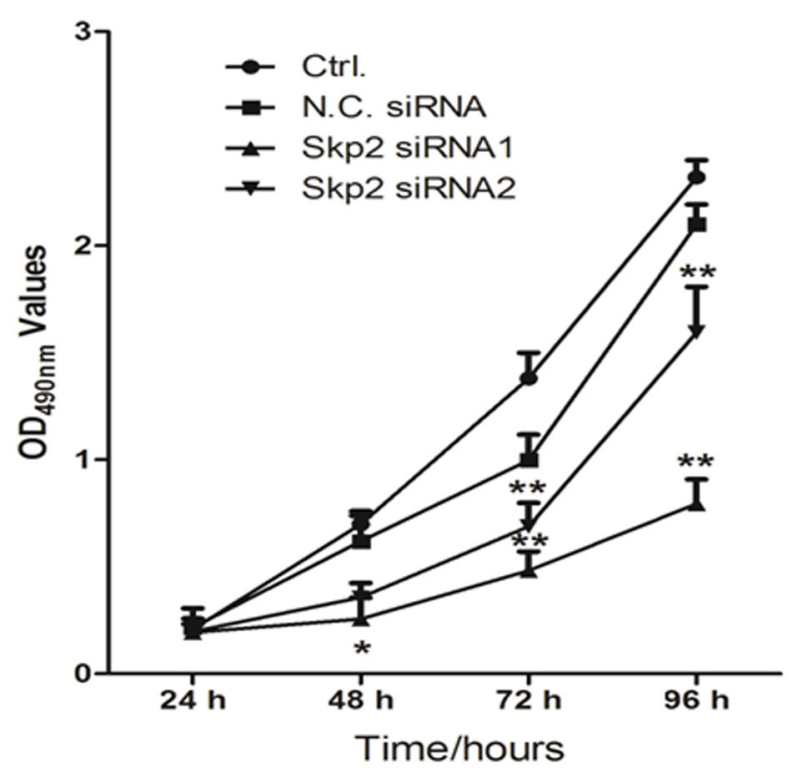

Figure 4. SKP2 siRNA inhibits MDA-MB-231 proliferation. After transfection with SKP2 siRNA1 or siRNA2 for 24, 48, 72, and $96 \mathrm{~h}$, a 3-(4,5-dimethylthiazol-2-yl)-2,5-diphenyltetrazolium bromide (MTT) assay was conducted to assess MDA-MB-231 proliferation capability. N.C. siRNA was used as a negative control. ${ }^{*} \mathrm{P}<0.05$ and ${ }^{* *} \mathrm{P}<$ 0.01 (compared with the N.C. siRNA group).

en (Kreiter et al., 2014). At present, cancer treatments include conventional surgery, radiation therapy, chemotherapy, hormone therapy, and cellular immune therapy (Hentschel et al., 2010; Antoine et al., 2014). Tumorigenesis is a complicated process that is affected by many things, such as genetics, microenvironment, and environmental factors. Activation or inactivation of multiple genes and cell proliferation signaling pathways such as PI3K/AKT, Wnt/ $\beta$-catenin, Src signaling pathways are involved. Therefore, finding a safe and effective gene therapy is one approach to cancer treatment.

The SKP2 gene is a newly discovered cancer gene that is overexpressed in many cancers such as in gastric, lung, colon, prostate, ovarian, and laryngeal cancers, and in glioma ( $\mathrm{Lu}$ et al., 2002; Takanami, 2005; Ben-Izhak et al., 2009; Shi et al., 2011; Wei et al., 2013). SKP2 is a member of the F-box protein family, which is necessary for DNA replication. It constitutes one of the subunits of the ubiquitin protein ligase complex SCF-SKP2 that participate in the ubiquitin-proteasome degradation pathway. Some studies have reported that the ubiquitin substrates of SKP2 include various kinds of cell cycle regulatory factors and transcription factors, such as cyclin A, cyclin B, cyclin E, cyclin D1, p21, p27, p53, p57, and E2F. Our results showed that SKP2 expression is significantly higher in patients with invasive breast cancer than in normal breasts. Previous research has suggested that SKP2 mainly degrades CKI p27 in the cell cycle, while high expression of SKP2 in malignant tumors is often accompanied by significantly reduced p27 (Pavlides et al., 2013; Chan et al., 2014). This is in agreement with our results.

siRNA gene silencing is an important gene function analysis method that is widely used in molecular studies (Nielsen et al., 2014). Specifically, siRNA interference can silence 
genes at the mRNA level. At the cell level, siRNA interference technology shows significant inhibitory effects. It is characterized by a shorter action cycle and fewer side-effects, and can silence multiple genes specifically with minimized side effects. In the present study, we designed and synthesized two pairs of SKP2-specific siRNA, siRNA1 and siRNA2, and detected the endogenous SKP2 expression inhibitory effect in breast cancer cells MDA-MB-231. Western blotting showed that SKP2specific siRNA1 can effectively inhibit endogenous SKP2 expression in breast cancer cells. Moreover, SKP2 silence significantly reduced breast cancer cell MDA-MB-231 proliferation. Therefore, we hypothesize that SKP2 might be a useful target for breast cancer gene therapy. Our study could provide an experimental basis and reference for breast cancer gene therapy.

\section{Conflicts of interest}

The authors declare no conflict of interest.

\section{ACKNOWLEDGMENTS}

We thank the anonymous reviewers for reviewing this manuscript.

\section{REFERENCES}

Al Joudi FS (2014). Human mammaglobin in breast cancer: a brief review of its clinical utility. Indian J. Med. Res. 139: 675-685.

Antoine C, Ameye L, Paesmans M and Rozenberg S (2014). Systematic review about breast cancer incidence in relation to hormone replacement therapy use. Climacteric 17: 116-132.

Ben-Izhak O, Akrish S, Gan S and Nagler RM (2009). SKP2 and salivary cancer. Cancer Biol. Ther. 8: 153-158.

Brown-Glaberman U, Dayao Z and Royce M (2014). HER2-targeted therapy for early-stage breast cancer: a comprehensive review. Oncology 28: 281-289.

Chan CH, Morrow JK, Zhang S and Lin HK (2014). SKP2: a dream target in the coming age of cancer therapy. Cell Cycle 13: 679680.

Chen L, Wu T, Wei TQ, Wei X, et al. (2014). SKP2-mediated degradation of p27 regulates cell cycle progression in compressed human bladder smooth muscle cells. Kaohsiung J. Med. Sci. 30: 181-186.

Chen Q, Xie W, Kuhn DJ, Voorhees PM, et al. (2008). Targeting the p27 E3 ligase SCF(SKP2) results in p27- and SKP2-mediated cell-cycle arrest and activation of autophagy. Blood 111: 4690-4699.

Cho J, Jung SY, Lee JE, Shim EJ, et al. (2014). A review of breast cancer survivorship issues from survivors' perspectives. J. Breast Cancer 17: 189-199.

Deapen D (2007). Breast implants and breast cancer: a review of incidence, detection, mortality, and survival. Plast. Reconstr. Surg. 120: 70S-80S

Goldner B, Behrendt CE, Schoellhammer HF, Lee B, et al. (2014). Incidence of inflammatory breast cancer in women, 1992-2009, United States. Ann. Surg. Oncol. 21: 1267-1270.

Hao B, Zheng N, Schulman BA, Wu G, et al. (2005). Structural basis of the Cks1-dependent recognition of p27(Kip1) by the SCF(SKP2) ubiquitin ligase. Mol. Cell 20: 9-19.

Hentschel S, Heinz J, Schmid-Höpfner S, Obi N, et al. (2010). The impact of menopausal hormone therapy on the incidence of different breast cancer types - data from the Cancer Registry Hamburg 1991-2006. Cancer Epidemiol. 34: 639-643.

Jiang D, Wang X, Liu X and Li F (2014). Gene delivery of cyclin-dependent kinase inhibitors p21Waf1 and p27Kip1 suppresses proliferation of MCF-7 breast cancer cells in vitro. Breast Cancer 21: 614-623.

Keramatinia A, Mousavi-Jarrahi SH, Hiteh M and Mosavi-Jarrahi A (2014). Trends in incidence of breast cancer among women under 40 in Asia. Asian Pac. J. Cancer Prev. 15: 1387-1390.

Kreiter E, Richardson A, Potter J and Yasui Y (2014). Breast cancer: trends in international incidence in men and women. $B r . J$. Cancer 110: 1891-1897.

Lu L, Schulz H and Wolf DA (2002). The F-box protein SKP2 mediates androgen control of p27 stability in LNCaP human prostate cancer cells. BMC Cell Biol. 3: 22.

Mamillapalli R, Gavrilova N, Mihaylova VT, Tsvetkov LM, et al. (2001). PTEN regulates the ubiquitin-dependent 
degradation of the CDK inhibitor p27(KIP1) through the ubiquitin E3 ligase SCF(SKP2). Curr. Biol. 11: 263-267.

Meads C and Moore D (2013). Breast cancer in lesbians and bisexual women: systematic review of incidence, prevalence and risk studies. BMC Public Health 13: 1127.

Nielsen C, Kjems J, Sørensen KR, Engelholm LH, et al. (2014). Advances in targeted delivery of small interfering RNA using simple bioconjugates. Expert Opin. Drug Deliv. 11: 791-822.

Njor S, Nyström L, Moss S, Paci E, et al. (2012). Breast cancer mortality in mammographic screening in Europe: a review of incidence-based mortality studies. J. Med. Screen 19: 33-41.

Pavlides SC, Huang KT, Reid DA, Wu L, et al. (2013). Inhibitors of SCF-SKP2/Cks1 E3 ligase block estrogen-induced growth stimulation and degradation of nuclear p27kip1: therapeutic potential for endometrial cancer. Endocrinology. 154: 4030-4045.

Polak P, Lawrence MS, Haugen E, Stoletzki N, et al. (2014). Reduced local mutation density in regulatory DNA of cancer genomes is linked to DNA repair. Nat. Biotechnol. 32: 71-75.

Shi P, Zhang Y, Tong X, Yang Y, et al. (2011). Dihydrotestosterone induces p27 degradation via direct binding with SKP2 in ovarian and breast cancer. Int. J. Mol. Med. 28: 109-114.

Shibahara T, Onishi T, Franco OE, Arima K, et al. (2005). Down-regulation of SKP2 is correlated with p27-associated cell cycle arrest induced by phenylacetate in human prostate cancer cells. Anticancer Res. 25: 1881-1888.

Solyom S, Aressy B, Pylkäs K, Patterson-Fortin J, et al. (2012). Breast cancer-associated Abraxas mutation disrupts nuclear localization and DNA damage response functions. Sci. Transl. Med. 4: 122ra23.

Stivala LA, Cazzalini O and Prosperi E (2012). The cyclin-dependent kinase inhibitor p21CDKN1A as a target of anticancer drugs. Curr. Cancer Drug Targets 12: 85-96.

Takanami I (2005). The prognostic value of overexpression of SKP2 mRNA in non-small cell lung cancer. Oncol. Rep. 13: 727-731.

Wei Z, Jiang X, Liu F, Qiao H, et al. (2013). Downregulation of SKP2 inhibits the growth and metastasis of gastric cancer cells in vitro and in vivo. Tumour Biol. 34: 181-192.

Zhang H, Kobayashi R, Galaktionov K and Beach D (1995). p19Skp1 and p45SKP2 are essential elements of the cyclin A-CDK2 S phase kinase. Cell 82: 915-925. 